\title{
4
}

\section{Malaria Transmission in the African Highlands in a Changing Climate Situation: Perspective from Kenyan Highlands}

\author{
Yaw A. Afrane1,2, Andrew K. Githeko ${ }^{1}$ and Guiyun Yan ${ }^{3}$ \\ ${ }^{1}$ Climate and Human Health Research Unit, \\ Centre for Global Health Research, Kenya Medical Research Institute; \\ ${ }^{2}$ School of Health Sciences, Bondo University College, Bondo; \\ ${ }^{3}$ Program in Public Health, College of Health Sciences, \\ University of California at Irvine, Irvine CA, \\ 1,2Kenya \\ ${ }^{3}$ U.S.A
}

\section{Introduction}

Vector-borne diseases are among the diseases that have been linked with climate change (IPCC. 2001). Malaria is probably the deadliest climate sensitive vector-borne disease (Githeko et al. 2000). About $90 \%$ of the $300-500$ million cases of the reported malaria cases worldwide come from Africa. In the late 80s to the 90s, malaria epidemics occurred frequently in western Kenya highlands, often taking the population by surprise. The epidemics were caused by Plasmodium falciparum and transmitted by Anopheles gambiae and Anopheles funestus. Epidemics were associated with high morbidity and mortality in all age groups, with prevalence of the disease rising from about $20 \%$ to about $60 \%$. The case mortality in functional health facilities were estimated at about 7.5\% (Githeko and Ndegwa 2001). The malaria transmission system involves a complex interaction between humans, mosquitoes, the plasmodium parasite, climate and the physical environment.

Warming of the climate is expected to lead to latitudinal and altitudinal temperature increase. The temporal and spatial changes in temperature, precipitation and humidity that are expected to occur under different climate change scenarios will affect the biology and ecology of vectors and intermediate hosts and consequently the risk of disease transmission. The risk increases because, although arthropods can regulate their internal temperature by changing their behaviour, they cannot do so physiologically and are thus critically dependent on climate for their survival and development (Lindsay and Birley 1996).

Temperature is inversely related to altitude, thus the high-elevation areas in Africa, or highlands, generally exhibit low ambient temperature, which restricts the development of vectors and parasites. Because malaria transmission rate is temperature-dependent, any factor that alters the temperature in the highland would reduce the duration of parasite development, larval development and increase the mosquito biting rates, and subsequently increase malaria transmission in the highlands. 
The greatest effect of climate change on transmission is likely to be observed at the extremes of the range of temperatures at which transmission occurs. For many diseases these lie in the range $14-18{ }^{\circ} \mathrm{C}$ at the lower end and $35-40{ }^{\circ} \mathrm{C}$ at the upper end. Warming in the lower range has a significant and non-linear impact on the extrinsic incubation period (Watts et al. 1987), and consequently disease transmission, while, at the upper end, transmission could cease. However, at around $30-32{ }^{\circ} \mathrm{C}$, vectorial capacity can increase substantially owing to a reduction in the extrinsic incubation period, despite a reduction in the vector's survival rate (Githeko et al. 2000).

Climatic conditions affect malaria transmission in a number of ways. The development rate of immature mosquitoes is very much temperature dependent. Below $16^{\circ} \mathrm{C}$, development of Anopheles gambiae, the main malaria vector in most parts of Africa, will completely stop, and the larvae will die in water temperature below $14^{\circ} \mathrm{C}$. In low-temperature conditions, mosquito larval development is severely delayed and high mortality incurs. In the adult stage, increase in ambient temperature will accelerate the digestion of blood meals taken by mosquitoes leading to increased human biting frequency and malaria transmission. While female mosquitoes obtain blood meal to develop their eggs, they pick up malaria parasites which they develop and become infectious to the next human host that is subsequently fed upon. Increased biting frequency and faster blood meal digestion also means increased fecundity and better reproductive fitness. Increased temperature also shortens the development time for the malaria parasite in the mosquito (Githeko et al. 2000).

Mosquito species such as the Anopheles gambiae complex and An. funestus are responsible for transmission of most of the malaria in the continent of Africa and are sensitive to temperature changes as immature stages in the aquatic environment and as adults. If water temperature rises, the larvae take a shorter time to mature (Munga et al. 2007) and consequently there is a greater capacity to produce more offspring during the transmission period. In warmer climates, adult female mosquitoes digest blood faster and feed more frequently (Afrane et al. 2005), thus increasing transmission intensity. Increased biting frequency and faster blood meal digestion also means increased fecundity and better reproductive fitness (Afrane et al. 2006). Similarly, malaria parasites complete extrinsic incubation within the female mosquito in a shorter time as temperature rises (Afrane et al. 2008) thereby increasing the proportion of infective vectors. Warming above $34{ }^{\circ} \mathrm{C}$ generally has a negative impact on the survival of vectors and parasites (Rueda et al. 1990).

In addition to the direct influence of temperature on the biology of vectors and parasites, changing precipitation patterns can also have short- and long term effects on vector habitats. Increased precipitation has the potential to increase the number and quality of breeding sites for mosquitoes and the density of vegetation, affecting the availability of resting sites (Githeko and Ndegwa 2001).

In addition to warming of the climate, environmental changes, such as deforestation, could increase local temperatures in the highlands, which in turn accelerates vector and parasite development leading to increased transmission. Deforestation is a common event in many regions of Africa. For example, Malava forest, a tropical rain forest in Kakamega district, has shrunk from $150 \mathrm{~km}^{2}$ in 1965 to $86 \mathrm{~km}^{2}$ in 1997 (FAO 1993). In the East African highlands, 2.9 million hectares of forest were cleared between 1981 and 1990, representing an $8 \%$ reduction in forest cover in one decade (Lindblade 2000). Land use and land cover changes may modify the temperature and relative humidity of malaria vector habitats in the highlands. It was demonstrated that in the southwestern highlands of Uganda, maximum and minimum temperatures were significantly higher in communities bordering cultivated 
swamps than in those near natural swamps (Lindblade 2000). These changes in regional climate and microclimatic conditions of mosquito habitats causes abundant changes of the existing mosquito species, and may make some areas permissive to the proliferation of new species. For instance, deforestation caused the introduction of An. gambiae into a habitat that was previously dominated by An. moucheti in Cameroon (Manga et al. 1995). In northern Brazil, An. marajoara, a species previously of minor importance, has become the principal malaria vector following changes in land use (Conn et al. 2002).

\section{Effect of microclimate changes due to deforestation on malaria transmission}

In many parts of the world, including Africa and South America, changes in land use or land cover, especially deforestation, have been linked to changes in the microclimate of the area in question. These changes in microclimate due to changes in land uses and cover has been linked to changes in malaria transmission. The changes in land use affect temperature, humidity and rainfall or precipitation patterns of the area. These also, goes to affect the microhabitat of malaria transmitting mosquitoes and the parasite they transmit, thus affecting malaria transmission in the area. The link between changes in land use and land cover and malaria transmission has been clearly established, which are highlighted and discussed below.

\subsection{Deforestation impact on malaria vectors: case studies from western Kenya highlands}

We conducted studies in western Kenya to assess the possible effect of deforestation on the microclimate and the subsequent effect on malaria transmission indices in the area. We measured indoor and outdoor temperature and humidity in the forested and deforested areas in the highlands. Entomological parameters such as the duration of gonotrophic cycle and biting frequency, reproductive fitness, survivorship and development of mosquitoes were studied. The sporogonic development of malaria parasites in the mosquito was also studied. Vectorial capacity which measures the potential rate of contact between infectious vectors and susceptible hosts was calculated from these parameters. It is the product of the vector density in relation to man, the proportion that bite man twice, and the expectation of the infective life span of the vector (MacDonald 1957; Garrett-Jones and Shidrawi, 1969).

We found that deforestation increased the indoor mean temperature by $1.8{ }^{\circ} \mathrm{C}$. Mean maximum and minimum temperatures were increased by $2.3^{\circ} \mathrm{C}$ and $1.5^{\circ} \mathrm{C}$ respectively. Outdoor temperatures were increased by $0.5^{\circ} \mathrm{C}$. The mean indoor relative humidity in the deforested area was about $22.6 \%$ lower than in the forest area during the dry season $(79.88 \%$ vs. 57.29\%) (Afrane et al. 2005; Afrane et al. 2006; Afrane et al. 2008; Afrane et al. 2007). Temperature and humidity were measured in houses in study sites that were originally forest but large portions of it have been deforested. Canopy coverage was over $60 \%$ in the forested area and less than $10 \%$ in the deforested area. Temperature and humidity were measured using $\mathrm{HOBO}^{\circledR}$ data loggers (Onset Computer Corporation, Bourne, Massachusetts, U.S.A.) which were placed inside all the human-habited experimental houses in the forested and deforested areas of the site. There were five experimental houses in each of the areas.

Since the general body functions of mosquitoes are temperature dependent, these small changes will have a biological significance on their life history characteristics. The mean 
minimum and maximum temperatures are very crucial for the development of mosquitoes and the parasite they transmit and by extension malaria transmission. Below $16^{\circ} \mathrm{C}$, development of many malaria vectors in Africa will completely stop, and the larvae will die in water temperature below $14^{\circ} \mathrm{C}$. The parasite in the mosquitoes has an upper and lower threshold of $32^{\circ} \mathrm{C}$ and $18^{\circ} \mathrm{C}$ respectively (Boyd 1949; MacDonald 1957).

The changes in the microclimate that were observed as a result of the deforestation caused a decrease in the duration of gonotrophic cycle of mosquitoes by 1.7 days (4.6 vs 2.9 days). The duration of gonotrophic cycle is the the period between the taking of a blood meal by a mosquito through digestion of the blood meal and oviposition or egg laying (Santos et al. 2002). The decreased duration of the gonotrophic cycles implies a decrease in human biting frequency from an average of once every 5 days to once every 3 days (Afrane et al. 2005). Increase in biting frequency means that the An. gambiae will feed more frequently on humans and in the process picks up infection, become infective and then transmit the parasite when they next bite another person.

The changes in the microclimate due to deforestation however, did not favour the survival of the adult An. gambiae. The effect of deforestation decreased median survival of An. gambiae by 5-7 days. The Anopheles gambiae mosquito rather prefers areas with high humidity. Since deforestation caused a decrease in the humidity, it rather caused a decrease in the survival of An. gambiae. However, despite the decreased survivorship of the mosquitoes due to the effects of deforestation, mosquitoes still had an enhanced reproductive fitness by $40 \%$. Over the course of their life span, mosquitoes in deforested areas laid far more eggs and thus, had better fecundity than mosquitoes in the forested areas by over $65 \%$ (Afrane et al. 2006). The reproductive fitness of a mosquito is its ability to reproduce over its entire life span. Fecundity, measured by the number of offspring a female mosquito can produce, is a major fitness trait (Hard et al. 1989.). The implication of these findings is that An. gambiae could increase its population within a short time when breeding sites are available. This could potentially lead to an increase in malaria transmission when infected humans are available.

The microclimatic changes also caused the parasite in the An. gambiae to have a reduced sporogonic development time from an average of 14 to 12.6 days (Afrane et al. 2008). Both oocyst and sporozoite development times were reduced by 1 and 1.4 days respectively. Reduced parasite development time in mosquitoes implies that the parasite will take a shorter time to become infective inside the mosquito and therefore will be transmitted faster from the mosquito to humans.

Vectorial capacity of the malaria vector was then calculated using the parameters that were measured in the studies described above.

$$
\text { Vectorial capacity }=\frac{m a^{2} p n}{\left(-\log _{\mathrm{e}} p\right)}
$$

Where $m$ is the relative density of vectors in relation to human, $a$ is the average number of humans bitten by one mosquito in one day, $p$ is the proportion of vectors surviving per day, and $n$ is the duration of sporogony in days. (MacDonald 1957).

This formula postulates that the length of the sporogonic development of malaria parasites in mosquitoes, biting frequency and vector survivorship are the most important factors for vector-borne malaria transmission 
We found that, deforestation substantially facilitated malaria transmission in the highland, as evidenced by two-fold increase in vectorial capacity (Afrane et al. 2008). These are shown in Table 1. Malaria is highly endemic in lowland areas. All the indices for malaria transmission were very much enhanced in the lowland site used for this study. This explains the huge difference between the lowland and two highland sites. In the highland sites, changes in the microclimate due to deforestation explain the changes in vectorial capacity of the two sites.

\begin{tabular}{ccccccc}
\hline Site & $\begin{array}{c}\text { Land use } \\
\text { type }\end{array}$ & $m$ & $a$ & $n$ & $P$ & $\begin{array}{c}\text { Vectorial } \\
\text { capacity }\end{array}$ \\
\hline Highland & Forested & 3.05 & 0.198 & 13.9 & 0.927 & 0.54 \\
& $\begin{array}{c}\text { Deforeste } \\
\mathrm{d}\end{array}$ & 4.64 & 0.233 & 12.8 & 0.917 & 0.96 \\
Lowland & $\begin{array}{c}\text { Deforeste } \\
\mathrm{d}\end{array}$ & 7.85 & 0.465 & 11.7 & 0.923 & 8.30 \\
\hline
\end{tabular}

Note.- $m$ is the relative density of vectors in relation to human,

$P$ is the proportion of vectors surviving per day,

$a$ is the average number of men bitten by one mosquito in one day, and

$n$ is the duration of sporogony in days.

Table 1. Estimated vectorial capacity of A. gambiae in forested and deforested areas in western Kenya highland and lowland.

\subsection{Land use and land cover changes and malaria transmission}

Land use and land cover changes have the potential to alter the micro-environment of malaria vectors which in turn affects their malaria transmission potential. In the highlands of Uganda, Lindblade and others (2000) compared mosquito density, biting rates, sporozoite rates and entomological inoculation rates between 8 villages located along natural papyrus swamps and 8 villages located along swamps that have been drained and cultivated. Since vegetation changes affect evapotranspiration patterns and, thus, local climate, they also investigated differences in temperature, humidity and saturation deficit between natural and cultivated swamps. They found that on average all malaria indices were higher near cultivated swamps. Maximum and minimum temperatures were significantly higher in communities bordering cultivated swamps. They found that the average minimum temperature of a village was significantly associated with the number of Anopheles gambiae s.l. per house. From their studies, it appears that replacement of natural swamp vegetation with agricultural crops led to increased temperatures, which may be responsible for elevated malaria transmission risk in cultivated areas.

To determine the effects of land cover types on mosquito productivity, Munga and others (2007) created semi-natural larval habitats within three land cover types (farmland, forest, and natural swamp) and filled them with Anopheles gambiae larvae. The mosquito pupation rate in farmland habitats was significantly greater than in swamp and forest habitats and larval-to pupal development times were significantly shorter. Land cover type may affect larval survivorship and adult productivity through its effects on water temperature and nutrients in the aquatic habitats. They found significantly higher water temperatures in farmland habitats than the other land cover types, which enhanced pupation rates and shortened development times. They also found a significant negative correlation of the occurrence of An. gambiae larvae with canopy cover and emergent plants 
in natural habitats. Their results suggest that deforestation and cultivation of natural swamps in the western Kenyan highland create conditions favorable for the survival of An. gambiae larvae, and consequently increase the risks of malaria transmission to the human population.

Other land cover changes such as swamp reclamation have been shown to have similar effects as deforestation on malaria vectors development. In other studies from western Kenya highlands, removal of swamp vegetation reduced shading and increased the breeding habitat water temperature by as much as $5{ }^{\circ} \mathrm{C}$ and whose effect reduced larval development time leading to increased vector productivity and abundance (Wamae et al. 2010). This has the potential to increase the population of mosquitoes and to which could also potentially cause an increase in malaria transmission if infected humans are available.

\section{Proliferation of mosquito species to new areas}

Deforestation has the potential to also change the microclimate of an area to become suitable to species that previously could not survive in the area. Each mosquito species has their own optimum survival conditions that allow them to survive or not to survive in an area. These survival conditions are mostly climate dependent. For instance, An. gambiae and Anopheles arabiensis, the most important vectors of human malaria in Africa are sibling species. An. arabiensis is either absent or shows a very low abundance in high-elevation areas where temperature is low and relative humidity and the amount of rainfall are high, but it is abundant in dry areas with less humidity which is the preferred habitat (Minakawa et al. 2002; Maharaj 2003). Studies were conducted by Afrane and others (2007) to investigate whether climate conditions in the western Kenya highlands were permissive to the development and survival of An. arabiensis and whether deforestation promoted $A n$. arabiensis survivorship of immature and adult stages, using life-table analysis.

It was found that $A n$. arabiensis larvae were able to survive in the deforested sites compared to the forested areas. Adult An. arabiensis placed in houses in the deforested area had an enhanced survival and better reproductive fitness than those in the forested area (Afrane et al. 2007). The implications of these findings are that, if the current trends of deforestation continue in the highlands, there could be a three vector system in the highlands, instead of the current two. This could potentially worsen the malaria transmission system in the highlands and could lead to the return of epidemics in the highlands. We expect that, environmental changes such as deforestation and global warming may facilitate the establishment of An. arabiensis populations in the highlands.

In the highland areas west to Mount Kenya which has an elevation of 1,720 - 1,921 m above sea level, malaria has been reported in the last 10 - 15 years. It was not clear whether these cases were introduced from the nearby lowland or resulted from local transmission because of no record of vector mosquitoes on the highlands. Chen and others (2006) reported finding mosquitoes breeding in the highlands. This means that the environment has become conducive for the proliferation of malaria vector species. Environmental changes and global warming could be a factor driving this emergence of malaria vectors to areas in mount Kenya region.

Manga and others (1995), working in an area that has been deforested to build a new airport in Cameroon, observed that deforestation caused the introduction of An. gambiae into a habitat that was previously predominated by An. moucheti. An. gambiae is the most ferocious of all the malaria vectors in Africa. It has been found to be the most efficient vector of $P$. 
falciparum in Africa. If the effects of deforestation made it possible for An. gambiae to inhabit this new place, the implications are that malaria transmissions in this area will most likely increase.

In northern Brazil, Conn and others (2002) found that An. marajoara, a species previously of minor importance, has become the principal malaria vector following changes in land use. Malaria transmission in the area was previously dominated by An. darlingi. However, land use and land cover changes made An. marajoara to become the most abundant vector and the most infected with malaria parasites, with its anthropophilic behavior. Their discovery highlights one of the challenges of neotropical malaria control, namely that the targeting of specific vectors may be complicated by a changing mosaic of different locally important vectors and their interactions with human populations.

Since 2003, Afrane and others (unpublished) have been studying the spatial and temporal dynamics of adult mosquitoes in the highlands of western Kenya. Each month, mosquitoes are collected from over 40 houses using pyrethrum spray catches. Mosquitoes are collected and brought to the laboratiories of the Kenya Medical Research Institute and identified to species using the morphological keys of Gilles and Demellion (1968). Polymerase Chain Reactions (PCRs) are done to discriminate the sibling species of An. gambiae complex.

Species composition reflects a combination of environmental and historical intervention events at a site; hence, changes in species composition can provide a sensitive measure of ecological changes. Figure 1 shows the changes in species composition of the An. gambiae s.l. complex from 2003 to 2010 in the study site. Analysis of species composition illustrated that the proportion of An. arabiensis in the site was characterized by a significant increase in the proportion of An. arabiensis from < 1\% in 2003 to 16\% in 2010.

\section{Iguhu}

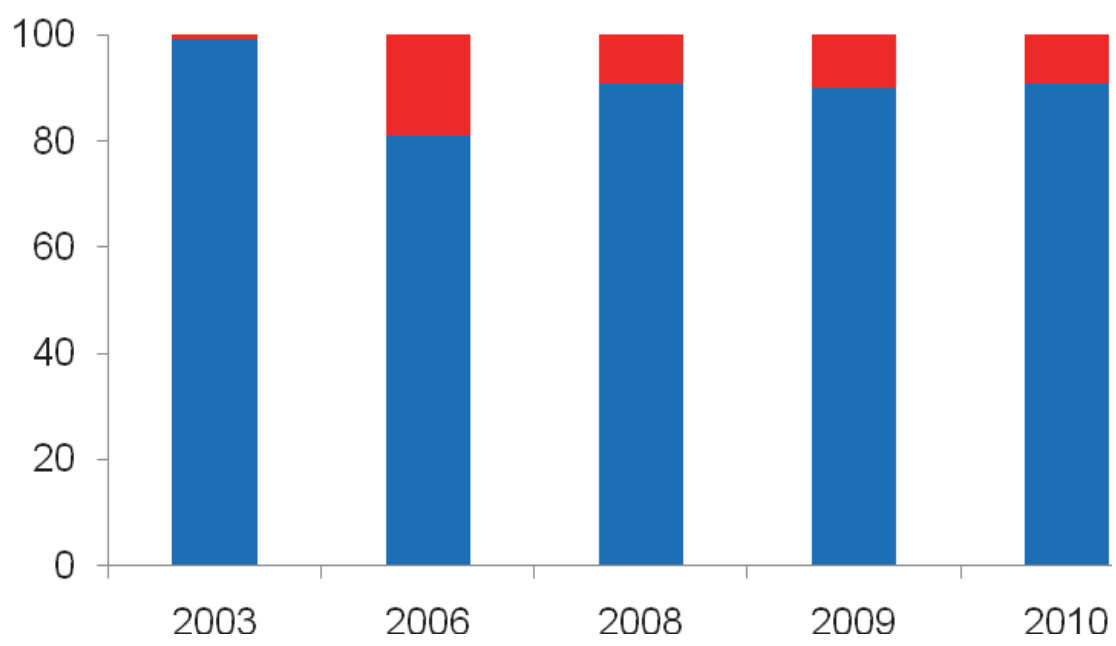

Fig. 1. Species composition of Anopheles gambiae (in blue) and An. arabiensis (in red) in Iguhu study site, Kakamega district in Kenya from 2003 to 2010. 


\section{Warming of the climate and malaria incidence in East Africa region}

Deforestation in the western Kenya highlands has permitted the gradual colonisation of $A n$. arabiensis into these areas that previously did not have this species. The implications are that with a new vector introduced in the area, the malaria transmission situation may worsen leading to resumption of epidemics in the site. The rate of malaria transmission is related to the abundance of malaria vectors while the stability of transmission is related to the diversity of species. Each species tends to dominate in the different seasons when its optimum survival conditions become available (Githeko et al. 2000).

There have been strong debates over the last decade by many authors on whether or not observed increases in malaria incidence in the Kenyan Highlands during the last thirty years are associated with co-varying changes in local temperature, possibly connected to global changes in climate. Studies using differing data sets and methodologies, produced conflicting results regarding the occurrence of temperature trends and their likelihood of being responsible, at least in part, for the increases in malaria incidence in the highlands of western Kenya. Omumbo and others (2011), used over 30 years of quality controlled daily observations of maximum, minimum and mean temperature in the analysis of trends at Kericho meteorological station, sited in Kenya's western highlands.

They found an upward trend of $\approx 0.2^{\circ} \mathrm{C} /$ decade in all three temperature variables. Mean temperature variations in the Kericho area were associated with large-scale climate variations including tropical Sea Surface Temperatures (SST). Local rainfall was found to have inverse effects on minimum and maximum temperature. They also used three versions of a spatially interpolated temperature data set, which showed markedly different trends when compared with each other and with their data.

Loevinsohn (1994) conducted a study that assessed the contribution of the climate to a malaria epidemic in Rwanda, focusing on the catchment area of one health centre where diagnosis was consistent and non-climatic variables well monitored. In late 1987 malaria incidence in the area increased by $337 \%$ over the 3 previous years. The increase was greatest in groups with little acquired immunity - children under 2 years $(564 \%)$ and people in highaltitude areas $(501 \%)$. Case-fatality rose significantly (relative risk $=4.85, \mathrm{p}<0.001$ ). The same year also saw record high temperatures and rainfall. An autoregressive equation including lagged effects of these two variables explained $80 \%$ of the variance in monthly malaria incidence. Temperature (especially mean minimum) predicted incidence best at higher altitudes where malaria had increased most. Empirically derived relations were consistent with the estimated generation time of the disease and with the known sensitivity of the plasmodium parasite to temperature. These findings are most relevant to regions near the altitude or latitude limits of the disease, where several epidemics have lately been reported.

\section{Commentary}

Forests have been shown to stabilize local temperatures and this can annul the effects of climate variability that is related to malaria epidemic in the highlands. Deforestation accounts for nearly $20 \%$ of global annual carbon emissions. Thus this phenomenon is closely linked to global warming and subsequent increased spread of malaria in the highlands. Forests are carbon sinks as they absorb $\mathrm{CO}_{2}$ and sequester it. Many policy makers are not familiar with the link between deforestation, climate change and their effect on malaria transmission. 
Changes in temperature which are as a result of environmental changes have the potential to affect the vector and their parasites as well as the transmission of many parasite borne diseases including malaria. Environmental changes, either natural phenomenon or through human intervention, alter the ecological balance and context within which vectors and their parasites breed, develop, and transmit disease (Patz et al. 2000). Small increases in temperature affect the mosquitoes and the parasites they transmit (Bruce-Chwatt 1987).

Large-scale conversion of tropical forests for agricultural purposes can significantly change the surface properties, such as soil wetness and surface roughness, of an area and could lead to changes in the local climate. For example, radiation budget and energy balance could be affected (Nobre et al. 1991; (Bounoua 2002). According to Ramakrishna and Running (Ramakrishna and Running 1995), the geometry of the new surface and albedo, which is a measure of the reflectivity of a surface, affect the radiation budget whilst the energy balance is also changed. This leads to a change in the microclimate of the area concerned. Ambient and indoor temperatures as well as humidity are affected by a change in land use (Lindblade 2000). For instance in the Amazon forest of South America, extensive deforestation caused a significant increase in the mean surface temperature (by about $2.5^{\circ} \mathrm{C}$ ) and a decrease in the annual evapo-transpiration (30\% reduction) and precipitation (25\% reduction) in the region, with the changes being more pronounced in the hot season (Nobre et al. 1991). The deforested area was associated with larger diurnal fluctuations of surface temperature and vapour pressure deficit. Land-cover changes that affect evaporation may also affect both the surface air temperature and the ground surface temperature in surrounding areas to a lesser extent (Skinner and Majorowicz 1999). In the tropics and subtropics, conversion warms canopy temperature by $0.8^{\circ} \mathrm{C}$ all year round (Bounoua 2002.). The data presented here have implications for understanding the effects of deforestation on an increased malaria transmission in the western Kenya and other African highlands. Malaria transmission force may be measured by the vectorial capacity of the vector. The duration of sporogony of $P$. falciparum in mosquitoes is exponentially related to the vectorial capacity (MacDonald 1957). If the daily survival and biting frequency of a vector are assumed to be constant, decreasing the duration of sporogony will lead to an increase in vectorial capacity. In this study deforestation led to a decrease in the duration of sporogony of $P$. falciparum by 1.1 days. This will translate into an exponential increase in vectorial capacity of An. gambiae which will lead to an increase in malaria transmission.

The studies presented here established the relationship between deforestation which is ongoing in the western Kenya highlands and the local climate as well as the effects of this altered local climate on malaria transmission indices in the study area.

The increase in indoor temperature due to land use and land cover changes enhanced digestion of blood meals by the mosquitoes in the deforested area leading to shorter gonotrophic cycles. Shorter gonotrophic cycles made mosquitoes to feed more frequently and thus increased their biting frequency. Frequent feeding by mosquitoes could increase the probability of picking infections from their hosts or infecting their hosts. Biting frequency affects the vectorial capacity and the Entomological Inoculation Rates (EIR), the two indices for measuring malaria transmission force in an area (Garrett-Jones and Shidrawi 1969). The vectorial capacity varies as the square of the daily man biting rates and EIR varies linearly with the daily man biting rates. An increase in the biting frequency of mosquitoes, thus, leads to an increase in vectorial capacity and EIR and hence to an increase in malaria transmission if there are infected individuals in the population. 
The present study also showed that land use and land cover changes increased the population of mosquitoes. The increased temperature as well as the decrease in humidity in the deforested site led to a higher reproductive fitness for mosquitoes. The intrinsic rate of growth which is the number of progeny per unit of time and thus more accurately reflects maximal fitness was increased by the effects of deforestation. Generation time for mosquitoes was also decreased in the deforested areas. Increase in the number of mosquitoes has implication for malaria transmission in an area where the effects of deforestation lead to increase in biting frequency. In the case where there are infected individuals in the population, increase in mosquito population and an increase in biting frequency will cause an increase in the EIR leading to an increase in malaria transmission.

In the deforested area $A n$. arabiensis had a shorter larva-to-adult development period. An. gambiae also exhibited the same phenomenon in a different study in the same area (Tuno et al. 2005). This renders the deforested area particularly vulnerable to mosquito proliferation and colonisation. The adults of An. arabiensis had a higher survival and reproductive fitness in the deforested area than in the forested areas. This could lead to proliferation in, and colonisation of the western Kenya highlands by An. arabiensis, a particularly crucial event since already An. gambiae and An. funestus are causing malaria epidemics in the highlands of western Kenya. Even though An. arabiensis is considered a zoophilic vector (prefers feeding on animals) rather than an anthropophilic vector (prefers feeding on humans), its efficiency to transmit malaria should not be underestimated. In many places in Africa such as the Kano plains and the lowland areas of western Kenya (White 1974; Githeko et al. 1996), Senegal (Robert et al. 1998) Ethiopia (Taye et al. 2005) and South Africa (Maharaj 2003), it is the main malaria vector. It is possible that it could become another vector in the highlands or it could also replace the current vectors in the highlands.

Deforestation had the effect of shortening the duration of the sporogony of P. falciparum. Time for the appearance of oocyst was shortened by 0.9 day and sporozoite appearance was also shortened by 1.1 days. This implies increased malaria transmission. The duration of sporogony of the malaria parasite in mosquitoes has an exponential relationship to the vectorial capacity of the vector. If all transmission variables remain the same, decreasing the duration of sporogony will cause an increase in the vectorial capacity. This translates into an increase in malaria transmission.

Besides their impacts on the duration of the gonotrophic cycle, biting frequency, duration of sporogony and subsequently on malaria transmission, deforestation and swamp reclamation also facilitate the creation of breeding habitats for An. gambiae. Those factors further enhance malaria transmission (Minakawa 2005; Munga et al. 2006). Mosquitoes will normally not breed in a natural swamp but will colonise a cultivated swamp (Munga et al. 2005). Deforestation opens up the forest, exposing the breeding habitats to direct sunlight which is a requirement for the maturation of the aquatic life stages of An. gambiae (Gilles and De Meillon 1968; Amerasinghe et al. 1997). As human populations increase in the highlands of western Kenya and elsewhere in the highlands of the East African region, deforestation and its subsequent effects on malaria transmission will continue.

The present study has shed some light on the possible impacts of increases in temperature in the highlands during periods of extreme events such as the El Nino events which are associated with anomalous warming and rainfall when they occur (Kovats et al. 2003; Rúa et al. 2005). In general a rise of $0.5^{\circ} \mathrm{C}$ in temperature in the highlands is equivalent to a decrease in altitude by $77 \mathrm{~m}$. This means highland climatic conditions move closer to 
lowland situations. The implication of this is that malaria transmission which is driven by temperature will also move closer to lowland situations.

Environmental changes such as deforestation and swamp reclamation have been shown to increase microclimate of an area. These changes in the microclimate have been shown to also affect the malaria mosquitoes and malaria transmission indices. Thus, if climate change should lead to altitudinal and latitudinal increases in temperature, this will go to affect malaria transmission especially in highaland areas of Africa.

The highlands of western Kenya are being impacted by three anthropogenic drivers of malaria transmission these being global warming, deforestation and swamp reclamation. Reforestation and swamp restoration can reverse increased local warming while providing, the local populations with ecological goods and services in a sustainable way. With regard to malaria such an intervention could potentially prevent the further spread of malaria to higher altitudes.

\section{Acknowledgements}

This study was supported by the National Institute of Health (R01 AI094580 , D43 TW01505 and R01 A150243).

\section{References}

Afrane, Y. A., B. W. Lawson, A. K. Githeko, and G. and Yan. 2005. Effects of Microclimatic Changes Due to Land use and Land Cover on the Duration of Gonotrophic Cycles of Anopheles gambiae Giles (Diptera: Culicidae) in Western Kenya Highlands. Journal of Medical Entomology 42: 974-980.

Afrane, Y. A., G. Zhou, B. W. Lawson, A. K. Githeko, and G. Yan. 2006. Effects of Microclimatic Changes Due to Deforestation on the Survivorship and Reproductive Fitness of Anopheles gambiae in Western Kenya Highlands. . Am. J., Trop Med Hyg. 74: 772-778.

Afrane, Y. A., T. Little, J., B. W. Lawson, A. K. Githeko, and G. Yan. 2008. Deforestation Increases the Vectorial Capacity of Anopheles gambiae Giles to Transmit Malaria in the Western Kenya Highlands. Emerg Infect Dis In Press.

Afrane, Y. A., G. Zhou, B. W. Lawson, A. K. Githeko, and G. Yan. 2007. Life-table analysis of Anopheles arabiensis in western Kenya highlands: effects of land covers on larval and adult survivorship. .Am J Trop Med Hyg. 77: 660-6.

Amerasinghe, F. P., F. Konradsen, K. T. Fonseka, and P. H. Amerasinghe. 1997. Anopheline (Diptera:Culicidae) breeding in a traditional tank-based village ecosystem in north central Sri Lanka. J Med Entomol. 34(290-7.

Bounoua, L., R. DeFries, G. J. Collatz, P. Sellers, and Khan, H. . 2002. Effects of land cover conversion on surface climate. Climatic Change 52: 29-64.

Boyd, M. F. 1949. Malariology. W. B. Saunders Co., Philadelphia.

Bruce-Chwatt, L. J. 1987. Malaria and its control: present situation and future prospects. Annu Rev Public Health 8: 75-110.

Chen, H., A. K. Githeko, G. Zhou, J. I. Githure, and G. Yan. 2006 New records of Anopheles arabiensis breeding on the Mount Kenya highlands indicate indigenous malaria transmission. Malar J. 7: 17. 
Conn, J. E., R. C. Wilkerson, M. N. Segura, R. T. de Souza, C. D. Schlichting, R. A. Wirtz, and M. M. Povoa. 2002. Emergence of a new neotropical malaria vector facilitated by human migration and changes in land use. Am J Trop Med Hyg 66: 18-22.

FAO. 1993. Forest resources assessment, 1990: Tropical countries. FAO forestry paper No.112, Rome, Italy

Garrett-Jones, C., and G. R. Shidrawi. 1969. Malaria vectorial capacity of a population of Anopheles gambiae: An exercise of epidemiological entomology. Bulletin of the World Health Organization 40: 531 - 545.

Gilles, M. T., and B. De Meillon. 1968. The Anopheline of Africa South of the Sahara. Publication of the South African Institute for Medical research no. 54.

Githeko, A. K., and W. Ndegwa. 2001. Predicting malaria epidemics in the Kenya highlands using climate data: a tool for decision makers. Global Change and Human Health 2: 54-63.

Githeko, A. K., S. W. Lindsay, U. E. Confalonieri, and J. A. Patz. 2000. Climate change and vector-borne diseases: a regional analysis. Bull World Health Organ 78: 1136-47.

Githeko, A. K., N. I. Adungo, D. M. Karanja, W. A. Hawley, J. M. Vulule, I. K. Seroney, A. V. Ofulla, F. K. Atieli, S. O. Ondijo, I. O. Genga, P. K. Odada, P. A. Situbi, and J. A. Oloo. 1996. Some observations on the biting behavior of Anopheles gambiae s.s., Anopheles arabiensis, and Anopheles funestus and their implications for malaria control. Exp Parasitol 82: 306-15.

Hard, J. J., W. E. Bradshaw, and D. J. Malarkey. 1989. Resource- and density-dependent development in treehole mosquitoes. Oikos 54: 137-144.

IPCC., and C. C. 2001. 2001. Impacts, Adaptations and Vulnerability. Contribution of Working Group 2 to the Third Assessment Report of the Intergovernmental Panel on Climate Change; . Cambridge University Press: Cambridge, UK and New York, NY, USA, ;: 1-970.

Kovats, R. S., M. J. Bouma, S. Hajat, E. Worrall, and A. Haines. 2003. El Nino and health. Lancet 362: 1481-9.

Lindblade, K. A., Walker E. D, Onapa A.W, Katungu J, and Wilson M, . . . . 2000. Land use change alters malaria transmission parameters by modifying temperature in a highland area of Uganda. Trop. Med. Int. Health 5: 263-74.

Lindsay, S. W. and M. H. Birley. 1996. Climate change and malaria transmission. Ann Trop Med Parasitol 90: 573-88.

Loevinsohn, M. E. 1994. Climatic warming and increased malaria incidence in Rwanda. Lancet 343: 714-718.

MacDonald, G. 1957. The Epidemiology and Control of Malaria. Oxford University Press, Oxford.

Maharaj, R. 2003. Life Table Characteristics of Anopheles arabiensis (Diptera: Culicidae) Under Simulated Seasonal Conditions. J. Med. Entomol. 40: 737-742.

Manga, L., J. C. Toto, and P. Carnevale. 1995. Malaria vectors and transmission in an area deforested for a new international airport in southern Cameroon. Societes Belges Medicine Tropicale 75: 43-49. 
Minakawa, N., G. Sonye, M. Mogi, A. Githeko, and G. Yan. 2002. The effects of climatic factors on the distribution and abundance of malaria vectors in Kenya. J Med Entomol 39: 833-41.

Minakawa, N., Munga, S., Atiel,.i F., Mushinzimana, E., Zhou, G, Githeko, A.K, Yan, G. 2005 Spatial distribution of anopheline larval habitats in Western kenyan highlands: effects of land cover types and topography. Am J Trop Med Hyg. 73: 157-65.

Munga, S., N. Minakawa, G. Zhou, A. K. Githeko, and G. Yan. 2007. Survivorship of immature stages of Anopheles gambiae s.l. (Diptera: Culicidae) in natural habitats in western Kenya highlands. J Med Entomol. 44: 758-64.

Munga, S., N. Minakawa, G. Zhou, E. Mushinzimana, O. J. Barrack, A. K. Githeko, and G. Yan. 2005. Association between land cover and habitat productivity of malaria vectors in western Kenya highlands. Am J Trop Med Hyg (in press).

Munga, S., N. Minakawa, G. Zhou, E. Mushinzimana, O. O. Barrack, A. K. Githeko, and Y. G. 2006. Association between land cover and habitat productivity of malaria vectors in western Kenyan highlands. Am J Trop Med Hyg 74: 69-75.

Nobre, C. A., P. J. Sellers, and J. Shukla. 1991. Amazonian deforestation and regional climate change. J. Clim 4: 957-988.

Omumbo, J. A., B. Lyon, S. M. Waweru, S. J. Connor, and M. C. Thomson. 2011. Raised temperatures over the Kericho tea estates: revisiting the climate in the East African highlands malaria debate. Malar J. 12.

Patz, J. A., T. K. Graczyk, N. Geller, and A. Y. Vittor. 2000. Effects of environmental change on emerging parasitic diseases. Int J Parasitol 30: 1395-405.

Ramakrishna, R., and S. W. Running. 1995. Satellite monitoring of global land cover changes and their impact on climate. Clim change 31: 315-493.

Robert, V., H. P. Awono-Ambene, and J. Thioulouse. 1998. Ecology of larval mosquitoes, with special reference to Anopheles arabiensis (Diptera: Culcidae) in marketgarden wells in urban Dakar, Senegal. . J. Med Entomol. . 35: 948-55.

Rúa, G. L., M. L. Quiñones, I. D. Vélez, J. S. Zuluaga, W. Rojas, G. Poveda, and D. Ruiz. 2005. Laboratory estimation of the effects of increasing temperatures on the duration of gonotrophic cycle of Anopheles albimanus (Diptera: Culicidae). Mem Inst Oswaldo Cruz, Rio de Janeiro 100: 515-520,.

Rueda, L. M., 1. K. J. Pate, R. C. Axtell, and R. E. Stinner, . 1990. Temperature-dependent development and survival rates of Culex quinquefasciatus and Aedes aegypti (Diptera: Culicidae). Journal of Medical Entomology 27: 892-898.

Santos, R. L., O. P. Forattini, and M. N. Burattini. 2002. Laboratory and field observations on duration of gonotrophic cycle of Anopheles albitarsis s.l. (Diptera: Culicidae) in southeastern Brazil. J Med Entomol. 39: 926-30. .

Skinner, W. R., and J. A. Majorowicz. 1999. regional climatic warming and associatd twentieth century land-cover changes in north western North America. Clim Res. 12: 39-52.

Taye, A., M. Hadis, N. Adugna, D. Tilahun, and R. A. Wirtz. 2005. Biting behavior and Plasmodium infection rates of Anopheles arabiensis from Sille, Ethiopia. . Acta Trop 16: ahead of print. 
Tuno, N., W. Okeka, N. Minakawa, M. Takagi, and G. Yan. 2005. Survivorship of Anopheles gambiae sensu stricto (Diptera: Culicidae) larvae in western Kenya highland forest. J Med Entomol 42: 270-7.

Wamae, P. M., A. K. Githeko, D. M. Menya, and W. Takken. 2010. Shading by Napier Grass Reduces Malaria Vector Larvae in Natural Habitats in Western Kenya Highlands. Ecohealth PMID: 20602147

Watts, D. M., D. S. Burke, B. A. Harrison, R. E. Whitmire, and A. Nisalak, . 1987. Effect of temperature on the vector efficiency of Aedes aegypti for dengue 2 virus. American Journal of Tropical Medicine and Hygiene 36: 143-152.

White, G. B. 1974. Anopheles gambiae complex and disease transmission in Africa. Trans R Soc Trop Med Hyg 68: 278-301. 


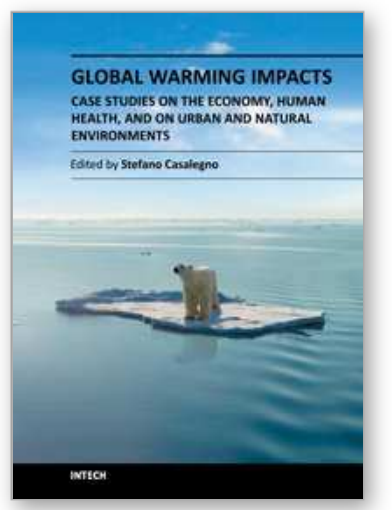

\author{
Global Warming Impacts - Case Studies on the Economy, Human \\ Health, and on Urban and Natural Environments \\ Edited by Dr. Stefano Casalegno
}

ISBN 978-953-307-785-7

Hard cover, 290 pages

Publisher InTech

Published online 03, October, 2011

Published in print edition October, 2011

This book addresses the theme of the impacts of global warming on different specific fields, ranging from the regional and global economy, to agriculture, human health, urban areas, land vegetation, marine areas and mangroves. Despite the volume of scientific work that has been undertaken in relation to each of each of these issues, the study of the impacts of global warming upon them is a relatively recent and unexplored topic. The chapters of this book offer a broad overview of potential applications of global warming science. As this science continues to evolve, confirm and reject study hypotheses, it is hoped that this book will stimulate further developments in relation to the impacts of changes in the global climate.

\title{
How to reference
}

In order to correctly reference this scholarly work, feel free to copy and paste the following:

Yaw A. Afrane, Andrew K. Githeko and Guiyun Yan (2011). Malaria Transmission in the African Highlands in a Changing Climate Situation: Perspective from Kenyan Highlands, Global Warming Impacts - Case Studies on the Economy, Human Health, and on Urban and Natural Environments, Dr. Stefano Casalegno (Ed.), ISBN: 978-953-307-785-7, InTech, Available from: http://www.intechopen.com/books/global-warming-impacts-casestudies-on-the-economy-human-health-and-on-urban-and-natural-environments/malaria-transmission-in-theafrican-highlands-in-a-changing-climate-situation-perspective-from-kenya

\section{INTECH}

open science | open minds

\author{
InTech Europe \\ University Campus STeP Ri \\ Slavka Krautzeka 83/A \\ 51000 Rijeka, Croatia \\ Phone: +385 (51) 770447 \\ Fax: +385 (51) 686166 \\ www.intechopen.com
}

\author{
InTech China \\ Unit 405, Office Block, Hotel Equatorial Shanghai \\ No.65, Yan An Road (West), Shanghai, 200040, China \\ 中国上海市延安西路65号上海国际贵都大饭店办公楼 405 单元 \\ Phone: +86-21-62489820 \\ Fax: +86-21-62489821
}


(C) 2011 The Author(s). Licensee IntechOpen. This is an open access article distributed under the terms of the Creative Commons Attribution 3.0 License, which permits unrestricted use, distribution, and reproduction in any medium, provided the original work is properly cited. 